\title{
PARALLELISABILITY OF PRINCIPAL FIBRE BUNDLES
}

\author{
BY \\ SHIING-SHEN CHERN AND SZE-TSEN HU
}

1. Introduction. One of the natural problems concerning fibre bundles is to determine whether or not a given fibre bundle $\mathfrak{F}=\left\{F, G ; X, B ; \psi, \phi_{U}\right\}$ is equivalent with the product bundle $B \times F$. It is proved in $[1, \S 1](1)$ that $\mathfrak{F}$ is equivalent with $B \times F$ if and only if its principal fibre bundle $\mathfrak{F}^{*}=\tau \mathfrak{F}$ is parallelisable; therefore, the problem posed above leads to the parallelisability of a given principal fibre bundle. For the notions and the notations used in the present paper, one may refer to those given by Shiing-shen Chern and Yi-fone Sun in the first section of their recent paper [1].

Throughout the present paper, let $\mathfrak{F}=\left\{G, G ; X, B ; \psi, \phi_{U}\right\}$ be a given principal fibre bundle in which the base space is a finite polyhedron. Let $B$ be given a triangulation such that the closure of every simplex is contained in some coordinate neighborhood $U$.

For each integer $n(0 \leqq n \leqq \operatorname{dim} B)$, let $B^{n}$ be the $n$-dimensional skeleton of $B$, that is, the set of simplexes of $B$ with dimensions not exceeding $n$. A principal fibre bundle $\mathfrak{F}$ is said to be $n$-parallelisable if there exists a mapping $f: B^{n} \rightarrow X$ such that $\psi f(b)=b$ for each point $b$ of $B^{n}$. In this case, $f$ is called an $n$-lifting of the principal fibre bundle $\mathfrak{F}$. Every principal fibre bundle $\mathfrak{F}$ is obviously 0 -parallelisable. Hence the problem posed above can be considered as solved if one has found a necessary and sufficient condition for an $(n-1)$ parallelisable principal fibre bundle $\mathfrak{F}$ to be $n$-parallelisable $(0<n \leqq \operatorname{dim} B)$. The object of the present paper is to give such a condition in terms of some cohomology invariants for the case that the reference group $G$ is pathwise connected.

2. Orientability of fibre bundles. A fibre bundle is said to be orientable if its principal fibre bundle is 1-parallelisable. For a pathwise connected reference group $G$, every fibre bundle is orientable. This is an immediate consequence of the following statement.

(2.1) If the reference group $G$ is pathwise connected, then every principal fibre bundle $\mathfrak{F}=\left\{G, G ; X, B ; \psi, \phi_{U}\right\}$ is 1-parallelisable.

Proof. Let $f: B^{0} \rightarrow X$ be an arbitrary 0 -lifting of $\mathfrak{F}$, the existence of which is obvious. According to our hypothesis with respect to the triangulation of $B$ made in $\$ 1$, for a given 1 -simplex $\sigma$ of $B$, there exists a coordinate neighborhood $U$ which contains the closure of $\sigma$. Let $a$ and $b$ be the two vertices of $\sigma$. Since $f(a) \in \psi^{-1}(a)$ and $f(b) \in \psi^{-1}(b)$, we might define two points $p, q \in G$ by taking

Presented to the Society, November 26, 1949; received by the editors December 10, 1948.

( $\left.{ }^{1}\right)$ The numbers in brackets denote the references in the bibliography at the end of the paper. 


$$
p=\phi_{U, a}^{-1} f(a), \quad q=\phi_{U, b}^{-1} f(b) .
$$

Since $G$ is pathwise connected, there exists a mapping $\theta: \mathrm{Cl} \sigma \rightarrow G$ such that $\theta(a)=p$ and $\theta(b)=q$. Extend the mapping $f$ into the interior of $\sigma$ by taking

$$
f(x)=\phi_{U}, x \theta(x) \quad(x \in \sigma) .
$$

Since $\psi \phi_{U, x} \theta(x)=x(x \in \sigma)$, this gives us a 1-lifting $f: B^{1} \rightarrow X$ of $\mathfrak{F}$ and (2.1) is proved.

3. The cocycle $w^{n}(f)$ of an $(n-1)$-lifting $f: B^{n-1} \longrightarrow X$. For the remainder of the paper, let $n$ be an integer such that $2 \leqq n \leqq \operatorname{dim} B$ and assume that the principal fibre bundle $\mathfrak{F}$ be $(n-1)$-parallelisable. Now, let $f: B^{n-1} \rightarrow X$ be an arbitrary $(n-1)$-lifting of $\mathfrak{F}$. We define a cochain $w^{n}(f)$ of $B$ with coefficients in the $(n-1)$ th homotopy group $\pi_{n-1}(G)$ of the reference group $G$ as follows.

Let $\sigma_{i}^{n}$ be an arbitrary $n$-simplex of $B$, then $f$ is defined on the boundary sphere $\partial \sigma_{i}^{n}$ of $\sigma_{i}^{n}$. According to our hypothesis concerning the triangulation of $B$, there exists a coordinate neighborhood $U$ which contains the closure $\mathrm{Cl} \sigma_{i}^{n}$ of $\sigma_{i}^{*}$. Since $f(b) \in \psi^{-1}(b)$ for each point $b$ of $\partial \sigma_{i}^{n}$, we might define a mapping $\theta: \partial \sigma_{i}^{n} \rightarrow G$ by taking

$$
\theta(b)=\phi_{U, b}^{-1} f(b) \quad\left(b \in \partial \sigma_{i}^{n}\right) .
$$

Since $G$ is $(n-1)$-simple [3, p. 69], $\theta$ determines a unique element $a_{i} \in \pi_{n-1}(G)$.

(3.1) The element $a_{i} \in \pi_{n-1}(G)$ depends only on the $(n-1)$-lifting $f: B^{n-1} \rightarrow X$ and the $n$-simplex $\sigma_{i}^{n} \in B$; hence the correspondence $\sigma_{i}^{n} \rightarrow a_{i}$ defines a cochain $w^{n}(f)$ of $B$ which depends only on $f$.

Proof. Let $V$ be another coordinate neighborhood which contains $\mathrm{Cl} \sigma_{i}^{n}$ and let $\theta^{\prime}: \partial \sigma_{i}^{n} \rightarrow G$ be the mapping defined by

$$
\theta^{\prime}(b)=\phi_{V, b}^{-1} f(b) \quad \cdot\left(b \in \partial \sigma_{i}^{n}\right) .
$$

It follows from the Paste Condition $[1, \S 1]$ for a principal fibre bundle that there exists an element $g \in G$ such that

$$
\theta^{\prime}(b)=g \cdot \theta(b) \quad\left(b \in \partial \sigma_{i}^{n}\right) .
$$

Since $G$ is pathwise connected, there exists a path $s: I \rightarrow G$ joining from the identity $e$ of $G$ to the element $g$, that is, $s(0)=e$ and $s(1)=g$. Define a homotopy $\theta_{t}: \partial \sigma_{i}^{n} \rightarrow G(0 \leqq t \leqq 1)$ by taking

$$
\theta_{t}(b)=s(t) \cdot \theta(b) \quad\left(b \in \partial \sigma_{i}^{n}, 0 \leqq t \leqq 1\right) .
$$

Then $\theta_{0}=\theta$ and $\theta_{1}=\theta^{\prime}$. Since $G$ is $(n-1)$-simple, $\theta^{\prime}$ determines the same element $a_{i} \in \pi_{n-1}(G)$ as $\theta$ does. This completes the proof of (3.1).

(3.2) The cochain $w^{n}(f)$ is a cocycle.

Proof. Let $\sigma^{n+1}$ be an arbitrary $(n+1)$-simplex of $B$. It need only be shown that 


$$
\left(\delta w^{n}(f)\right)\left(\sigma^{n+1}\right)=0,
$$

where $\delta w^{n}(f)$ denotes the coboundary of $w^{n}(f)$. According to our hypothesis regarding the triangulation of $B$, there exists a coordinate neighborhood $U$ which contains the closure $\mathrm{Cl} \sigma^{n+1}$ of $\sigma^{n+1}$. Let

$$
A^{n-1}=B^{n-1} \cap \mathrm{Cl} \sigma^{n+1}
$$

and define a mapping $\xi: A^{n-1} \rightarrow G$ by taking

$$
\xi(b)=\phi_{U, b}^{-1} f(b)
$$

According to S. Eilenberg [2, p. 237], $\xi$ determines an $n$-cocycle $c^{n}(\xi)$ of $\mathrm{Cl} \sigma^{n+1}$ with coefficients in $\pi_{n-1}(G)$. Clearly we have

$$
c^{n}(\xi)=w^{n}(f) \mid \mathrm{Cl} \sigma^{n+1} ;
$$

and hence

$$
\left(\delta w^{n}(f)\right)\left(\sigma^{n+1}\right)=\left(\delta c^{n}(\xi)\right)\left(\sigma^{n+1}\right)=0,
$$

that is, $w^{n}(f)$ is a cocycle. q.e.d.

4. The characteristic coset $W^{n}(\mathfrak{F})$. According to (3.1) and (3.2), every $(n-1)$-lifting $f: B^{n-1} \rightarrow X$ of $\mathfrak{F}$ determines an $n$-cocycle $w^{n}(f)$ of $B$ and hence an element $\omega^{n}(f)$ of the cohomology group $H^{n}\left(B, \pi_{n-1}(G)\right)$, called an $n$-dimensional obstruction element of the $(n-1)$-parallelisable principal fibre bundle $\mathfrak{F}$. The object of the present section is to prove that the $n$-dimensional obstruction elements of $\mathfrak{F}$ form a coset of the presentable subgroup $P^{n}\left(B, \pi_{n-1}(G)\right)$ in the cohomology group $H^{n}\left(B, \pi_{n-1}(G)\right)[4, \S 3]$.

(4.1) Every pair of $(n-1)$-liftings $f, g: B^{n-1} \rightarrow X$ of $\mathfrak{F}$ determines a unique mapping $\mu: B^{n-1} \rightarrow G$ denoted by $\mu=f^{-1} \cdot g$.

Proof. The required mapping $\mu: B^{n-1} \rightarrow G$ is defined as follows: For an arbitrary point $b \in B^{n-1}$, choose a coordinate neighborhood $U$ containing $b$ and define

$$
\mu(b)=\left(\phi_{U, b}^{-1} f(b)\right)^{-1} \cdot\left(\phi_{U, b}^{-1} g(b)\right) .
$$

To justify this definition, let $V$ be another coordinate neighborhood which contains $b$. By the aid of the Paste Condition $[1, \S 1]$, that $\phi_{V, b}^{-1} \phi_{U, b}$ is a left translation of $G$ determined by some element $\xi$ of $G$, one may easily verify that

$$
\begin{aligned}
\left(\phi_{V, b}^{-1} f(b)\right)^{-1} \cdot\left(\phi_{V, b}^{-1} g(b)\right) & =\left(\xi \cdot \phi_{U, b}^{-1} f(b)\right)^{-1}\left(\xi \cdot \phi_{U, b g}^{-1}(b)\right) \\
& =\left(\phi_{U, b}^{-1} f(b)\right)^{-1} \cdot \xi^{-1} \cdot \xi \cdot\left(\phi_{U, b}^{-1} g(b)\right)=\mu(b) .
\end{aligned}
$$

Hence the transformation $\mu$ is uniquely defined. The continuity of $\mu$ follows from the fact that $\mu$ is continuous in every coordinate neighborhood $U$. This completes the proof.

(4.2) Given an $(n-1)$-lifting $f: B^{n-1} \rightarrow X$ of $\mathfrak{F}$ and a mapping $\mu: B^{n-1} \rightarrow G$, 
there exists a unique $(n-1)$-lifting $g: B^{n-1} \rightarrow X$ of $\mathfrak{F}$ such that $f^{-1} \cdot g=\mu$.

Proof. The required $(n-1)$-lifting $g: B^{n-1} \rightarrow X$ is defined as follows: For an arbitrary point $b \in B^{n-1}$, choose a coordinate neighborhood $U$ which contains $b$ and define

$$
g(b)=\phi_{U, b}\left(\phi_{U, b}^{-1} f(b) \cdot \mu(b)\right) .
$$

To justify this definition, let $V$ be another coordinate neighborhood which contains $b$; then we have

$$
\begin{aligned}
\phi_{V, b}\left(\phi_{V, b}^{-1} f(b) \cdot \mu(b)\right) & =\phi_{U, b} \phi_{U, b}^{-1} \phi_{V, b}\left(\phi_{V, b}^{-1} \phi_{U, b} \phi_{U, b}^{-1} f(b) \cdot \mu(b)\right) \\
& =\phi_{U, b}\left(\xi^{-1} \cdot\left(\xi \cdot \phi_{U, b}^{-1} f(b) \cdot \mu(b)\right)=g(b),\right.
\end{aligned}
$$

where $\xi \in G$ has the same meaning as in the proof of $(4.1)$. Hence $g(b)$ is uniquely defined. The continuity of $g$ follows from the fact that $g$ is continuous in every coordinate neighborhood $U$. Further, clearly we have

$$
\psi g(b)=\psi \phi_{U, b}\left(\phi_{U, b}^{-1} f(b) \cdot \mu(b)\right)=b ;
$$

hence $g$ is an $(n-1)$-lifting of $\mathfrak{F}$. That $f^{-1} \cdot g=\mu$ is obvious. This completes the proof of (4.2).

(4.3) TheORem I. The totality of the n-dimensional obstruction elements of an $(n-1)$-parallelisable principal fibre bundle $\mathfrak{F}$ forms a coset $W^{n}(F)$ of the presentable subgroup $P^{n}\left(B, \pi_{n-1}(G)\right)$ in the cohomology group $H^{n}\left(B, \pi_{n-1}(G)\right)$, that is, $W^{n}(\mathfrak{F})$ is an element of the quotient group

$$
Q^{n}\left(B, \pi_{n-1}(G)\right)=H^{n}\left(B, \pi_{n-1}(G)\right) / P^{n}\left(B, \pi_{n-1}(G)\right) .
$$

Proof. Let $W^{n}(\mathfrak{F})$ denote the totality of the $n$-dimensional obstruction elements of $\mathfrak{F}$. First, let $f, g: B^{n-1} \rightarrow X$ be two arbitrary $(n-1)$-liftings of $\mathfrak{F}$ and let $\mu=f^{-1} \cdot g . \mu$ presents a presentable element $[4, \S 3]$ of $H^{n}\left(B, \pi_{n-1}(G)\right)$ represented by the cocycle $c^{n}(\mu)$, introduced by S. Eilenberg [2, p. 237]. Let $\sigma^{n}$ be an arbitrary $n$-simplex of $B$. According to our hypothesis concerning the triangulation of $B$, there exists a coordinate neighborhood $U$ which contains the closure $\mathrm{Cl} \sigma^{n}$ of $\sigma^{n}$. Then, by the construction given in the proof of (4.1), we have

$$
\mu(b)=\left(\phi_{U, b}^{-1} f(b)\right)^{-1} \cdot\left(\phi_{U, b}^{-1} g(b)\right) \quad\left(b \in \partial \sigma^{n}\right) .
$$

Hence, it follows from a homotopy property of a topological group [3] that

$$
c^{n}(\mu) \cdot \sigma^{n}=w^{n}(g) \cdot \sigma^{n}-w^{n}(f) \cdot \sigma^{n} .
$$

Hence it follows that

$$
w^{n}(g)=w^{n}(f)+c^{n}(\mu),
$$

and it implies that the obstruction elements $\omega^{n}(f)$ and $\omega^{n}(g)$ are contained in 
the same coset of $P^{n}\left(B, \pi_{n-1}(G)\right)$ in $H^{n}\left(B, \pi_{n-1}(G)\right)$. This proves that $W^{n}(\mathfrak{F})$ is contained in a single coset of the presentable subgroup $P^{n}\left(B, \pi_{n-1}(G)\right)$.

Conversely, let $f: B^{n-1} \rightarrow X$ be a given $(n-1)$-lifting of $\mathfrak{F}$ and let $\alpha$ be an arbitrary presentable element of $H^{n}\left(B, \pi_{n-1}(G)\right)$. According to the definition of presentable elements $[4, \S 3]$, there is a mapping $\mu: B^{n-1} \rightarrow G$ such that the cocycle $c^{n}(\mu)$ represents $\alpha$. Let $g: B^{n-1} \rightarrow X$ be the $(n-1)$-lifting of $\mathfrak{F}$ constructed in (4.2). Then it follows just as above that

$$
w^{n}(g)=w^{n}(f)+c^{n}(\mu) .
$$

This implies that $\omega^{n}(g)=\omega^{n}(f)+\alpha$. Hence, every element of the coset $\omega^{n}(f)+P^{n}\left(B, \pi_{n-1}(G)\right)$ is an obstruction element of $\mathfrak{F}$. This completes the proof of Theorem I.

5. $n$-Parallelisability theorems. We are now in a position to prove the main result of this paper.

(5.1) Theorem II. An $(n-1)$-parallelisable principal fibre bundle $\mathfrak{F}$ is $n$-parallelisable if and only if $W^{n}(\mathfrak{F})$ is the presentable subgroup $P^{n}\left(B, \pi_{n-1}(G)\right)$ of the cohomology group $H^{n}\left(B, \pi_{n-1}(G)\right)$.

Proof. Necessity. Suppose $\mathfrak{F}$ to be a $n$-parallelisable. Then there is an $n$-lifting $f^{*}: B^{n} \rightarrow X$ of $\mathfrak{F}$. Let $f=f^{*} \mid B^{n-1}$, then $f$ is an $(n-1)$-lifting with $w^{n}(f)=0$. It follows from (4.3) that $W^{n}(\mathfrak{F})=P^{n}\left(B, \pi_{n-1}(G)\right)$.

Sufficiency. Suppose that $W^{n}(\mathfrak{F})=P^{n}\left(B, \pi_{n-1}(G)\right)$. Then there exists an $(n-1)$-lifting $f: B^{n-1} \rightarrow X$ of $\mathfrak{F}$ such that its obstruction element $\omega^{n}(f)=0$, that is, the cocycle $w^{n}(f)$ is a coboundary. According to S. Eilenberg [2, (11.6)], there exists a mapping $\mu: B^{n-1} \rightarrow G$ such that $c^{n}(\mu)=-w^{n}(f)$. Let $g: B^{n-1} \rightarrow X$ be the $(n-1)$-lifting given in (4.2); then we have

$$
w^{n}(g)=w^{n}(f)+c^{n}(\mu)=w^{n}(f)-w^{n}(f)=0 .
$$

Let $\sigma_{i}^{n}$ be an arbitrary $n$-simplex of $B$. Choose a coordinate neighborhood $U$ which contains $\mathrm{Cl} \sigma_{i}^{n}$. Define a mapping $\theta_{i}: \partial \sigma_{i}^{n} \rightarrow G$ by taking

$$
\theta_{i}(b)=\phi_{U, b}^{-1} g(b) \quad\left(b \in \partial \sigma_{i}^{n}\right) .
$$

Since $w^{n}(g)=0, \theta_{i}$ has an extension $\theta_{i}^{*}: \mathrm{Cl} \sigma_{i}^{n} \rightarrow G$. Define a mapping $h_{i}: \mathrm{Cl} \sigma_{i}^{n}$ $\rightarrow X$ by taking

$$
h_{i}(b)=\phi_{U, b} \theta_{i}^{*}(b) \quad\left(b \in \mathrm{Cl} \sigma_{i}^{n}\right) .
$$

Then $h_{i}(b)=g(b)$ for each $b \in \partial \sigma_{i}^{n}$. Define a mapping $g^{*}: B^{n} \rightarrow X$ by taking

$$
g^{*}(b)=\left\{\begin{array}{lr}
g(b) & \left(b \in B^{n-1}\right), \\
h_{i}(b) & \left(b \in \sigma_{i}\right) .
\end{array}\right.
$$

Clearly, $g^{*}$ is an $n$-lifting of $\mathfrak{F}$. This completes the proof of Theorem II.

As an alternative form of Theorem II, we give the following statement. 
(5.2) Let $\mathfrak{F}$ be an $(n-1)$-parallelisable principal fibre bundle and $f: B^{n-1} \rightarrow X$ be a given $(n-1)$-lifting of $\mathfrak{F}$. $\mathfrak{F}$ is $n$-parallelisable if and only if the cocycle $w^{n}(f)$ be presentable.

The following theorem is an immediate consequence of Theorem II.

(5.3) THEOREM III. If $G$ is pathwise connected and

$$
H^{n}\left(B, \pi_{n-1}(G)\right)=P^{n}\left(B, \pi_{n-1}(G)\right)
$$

for each $2 \leqq n \leqq \operatorname{dim} B$, then every fibre bundle $\mathfrak{F}=\left\{F, G ; X, B ; \psi, \phi_{U}\right\}$ is equivalent with the product bundle $B \times F$.

\section{BIBLIOGRAPHY}

1. S. S. Chern and Y. F. Sun, The imbedding theorem for fibre bundles, Trans. Amer. Math. Soc. vol. 67 (1949) pp. 284-301.

2. S. Eilenberg, Cohomology and continuous mappings, Ann. of Math. vol. 41 (1940) pp. 231-251.

3. S. T. Hu, Some homotopy properties of topological groups and homogeneous spaces, Ann. of Math. vol. 49 (1948) pp. 67-74.

4. - Extension and classification of the mappings of a finite complex into a topological group or an n-sphere, Ann. of Math. vol. 50 (1949) pp. 158-173.

Academia Sinica,

Nanking, China. 